Notfall Rettungsmed 2009 · 12:575-575

DOI 10.1007/s10049-009-1236-8

Online publiziert: 5. Dezember 2009

(c) Springer-Verlag 2009
T. Nicolai ${ }^{1} \cdot$ M. Weiss ${ }^{2} \cdot$ U. Kreimeier ${ }^{3}$

${ }^{1}$ Dr. von Haunersches Kinderspital, Ludwig-Maximilians-Universität, München

${ }^{2}$ Anästhesieabteilung, Universitäts-Kinderkliniken, Zürich

${ }^{3}$ Klinik für Anaesthesiologie, Klinikum der Universität München

\title{
Notfälle beim Kind
}

Notfälle bei Kindern machen nur einen kleinen Anteil der Notarzteinsätze aus. Daher sind sie ungewohnte, nicht in die üblichen Routinen einzuordnende, Ereignisse. Zudem stellt ein in Not geratenes Kind für die meisten Rettungsmannschaften eine besondere emotionale Situation dar, die ausgesprochen stressbesetzt sein kann. Alleine die Einschätzung der klinischen Situation des Patienten kann hier ohne ständige Übung im Umgang mit kranken Kindern erschwert sein. Die Krankheitsbilder weichen meist völlig von den in der Erwachsenennotfallmedizin gewohnten ab und das Instrumentarium ist anders. Nicht zu vergessen ist ein wesentlicher Unterschied in der Versorgung von Kindern im Vergleich zu Erwachsenen: Kinder sind häufig nicht zur Kooperation fähig, eine Eigenanamnese muss durch die Befragung der Eltern ersetzt werden und die psychologische Komponente (ruhiges Auftreten, Sprechen) ist in der Versorgung von pädiatrischen Notfallpatienten oft mindestens ebenso wichtig wie Sauerstofftherapie und andere Maßnahmen.

\section{() Ohne Übung im Umgang mit kranken Kindern ist die Einschätzung der klinischen Situation häufig erschwert}

Gerade diese Umstände lassen es wünschenswert erscheinen, klare Ablaufschemata, brauchbare Techniken und möglichst einfache Hilfsmittel zu entwickeln, um die Versorgung von Kindern zu vereinfachen. Die Weiterbildung der Notärzte in für die Versorgung von Kindern wichtigen Themenbereichen hat sich die Deutsche Interdisziplinäre Vereinigung für Intensiv- und Notfallmedizin (DIVI) durch die Verabschiedung eines diesbezüglichen Curriculums zum besonderen Anliegen gemacht.
Die Ursache von lebensbedrohlichen Notfällen beim Kind ist häufig in der Respiration zu finden. Kleine Atemwegslumina, eingeschränkte respiratorische Reserven bei erhöhtem Sauerstoffverbrauch, hohe Infekthäufigkeit mit Zuschwellen der Atemwege, eine instabile Atemregulation beim Neugeborenen und erst allmählich sich verfestigende Rippenknorpel und aufbauende Atemmuskulatur bilden die physiologischen Grundlagen für diese gefährliche Kausalkette. Das Kind mit einem schweren Kruppanfall behält die vom Arzt oder Rettungsassistenten vorgehaltene Sauerstoff-Maske nicht auf dem Gesicht, bzw. erregt sich dabei so sehr, dass der Effekt ins Gegenteil verkehrt wird. Daher ist die Beherrschung respiratorischer Notfälle einschließlich des Atemwegsmanagements entscheidend in der Erstversorgung von Kindernotfällen. Die diesbezüglichen aktuellen Entwicklungen $(\mathrm{Maß}$ nahmen bei schwieriger Intubation, Larynxmaske bzw. Larynxtubus, geblockte Tuben auch für Kinder, Intubationshilfsmittel anderer Art) werden in diesem Heft dargestellt und bewertet.

\section{() Quantitativ geringe Blutverluste können bei Kindern zu ausgeprägten Kreislaufreaktionen führen}

Andere Unterschiede in der Versorgung von Kindernotfällen bestehen in der schwierigen Einschätzung des Intravasalvolumenstatus von Kindern mit Volumenmangel: einerseits wird dieser oft lange kompensiert (Brechdurchfallerkrankungen), andererseits erfolgt dann die Dekompensation umso fulminanter. Darüber hinaus muss der Notarzt darauf achten, dass selbst quantitativ geringe - und aus der Erwachsenenmedizin belanglos erscheinende - Blutverluste bei Kin- dern zu ausgeprägten Kreislaufreaktionen führen können - je kleiner die Patienten sind, desto relevanter ist hier eine Fehleinschätzung. Die Frühtherapie der Meningokokkensepsis wurde durch die aggressive Volumensubstitution schon in der präklinischen Phase revolutioniert: wegen der bekannten Schwierigkeit des Gefäßzuganges besonders für den pädiatrisch Ungeübten haben hier Techniken wie die längst etablierte intraossäre Punktionstechnik Fortschritte gebracht. Auch hier gibt es relevante technische Neuerungen, von denen im Leitthema berichtet wird.

Wir möchten mit dieser Ausgabe von „Notfall + Rettungsmedizin“ einen Einblick in die dynamischen Entwicklungen der Versorgung von Kindernotfällen vermitteln, den Leser für dieses Thema wieder sensibilisieren und zum Besuch von Trainingskursen etc. anregen.

\section{Muers}

T. Nicolai

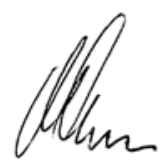

M Weiss

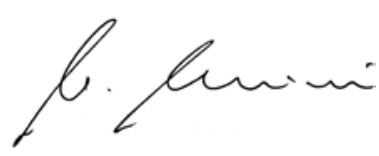

U Kreimeier

\section{Korrespondenzadresse}

Prof. Dr. T. Nicolai

Dr. von Haunersches Kinderspital, Ludwig-Maximilians-Universität Lindwurmstraße 4, 80337 München

thomas.nicolai@med.uni-muenchen.de 